\title{
Article
}

\section{Ultrafast High-Voltage Kicker System for Ion-Clearing Gaps}

\author{
Alexander Yu. Smirnov ${ }^{1, *}$, Ronald Agustsson ${ }^{1}$, Stephen V. Benson ${ }^{2}{ }^{\oplus}$, Dmitry Gavryushkin ${ }^{1}$, Jiquan Guo ${ }^{2}$, \\ Sergey V. Kutsaev ${ }^{1}{ }^{1}$, Adam Moro ${ }^{1}$, Gunn Tae Park ${ }^{2}$, Alexei V. Smirnov ${ }^{1,+}$, Haipeng Wang ${ }^{2}$ and Shukui Zhang ${ }^{2}$ \\ 1 RadiaBeam Technologies, LLC, Santa Monica, CA 90404, USA; agustsson@radiabeam.com (R.A.); \\ dmitriyg@radiabeam.com (D.G.); kutsaev@radiabeam.com (S.V.K.); amoro@radiabeam.com (A.M.); \\ asmirnov@viewray.com (A.V.S.) \\ 2 Thomas Jefferson National Accelerator Facility, Newport News, VA 23606, USA; felman@jlab.org (S.V.B.); \\ jguo@jlab.org (J.G.); gpark@jlab.org (G.T.P.); haipeng@jlab.org (H.W.); shukui@jlab.org (S.Z.) \\ * Correspondence: alexander.smirnov@radiabeam.com; Tel.: +1-310-822-5845 \\ † Current address: ViewRay Inc., Mountain View, CA 94043, USA.
}

Citation: Smirnov, A.Y.; Agustsson,

R.; Benson, S.V.; Gavryushkin, D.;

Guo, J.; Kutsaev, S.V.; Moro, A.; Park, G.T.; Smirnov, A.V.; Wang, H.; et al.

Ultrafast High-Voltage Kicker System for Ion-Clearing Gaps. Photonics 2021, 8, 507. https://doi.org/10.3390/ photonics 8110507

Received: 14 September 2021 Accepted: 9 November 2021 Published: 11 November 2021

Publisher's Note: MDPI stays neutral with regard to jurisdictional claims in published maps and institutional affiliations.

Copyright: (c) 2021 by the authors. Licensee MDPI, Basel, Switzerland. This article is an open access article distributed under the terms and conditions of the Creative Commons Attribution (CC BY) license (https:// creativecommons.org/licenses/by/ $4.0 /)$.

\begin{abstract}
Ionization scattering of electron beams with residual gas molecules causes ion trapping in electron rings, both in a collider and electron cooling system. These trapped ions may cause emittance growth, tune shift, halo formation, and coherent coupled bunch instabilities. In order to clear the ions and prevent them from accumulating turn after turn, the gaps in a temporal structure of the beam are typically used. Typically, the gap in the bunch train has a length of a few percent of the ring circumference. In those regions, the extraction electrodes with high pulsed voltages are introduced. In this paper, we present the design consideration and initial test results of the high-voltage pulsed kicker hardware that includes vacuum device and pulsed voltage driver, capable of achieving over $3 \mathrm{kV}$ of deflecting voltage amplitude, rise and fall times of less than $10 \mathrm{~ns}, 100 \mathrm{~ns}$ flat-top duration at $1.4 \mathrm{MHz}$ repetition rate.
\end{abstract}

Keywords: electron-ion collider; beam kicker; high voltage; electron cooling

\section{Introduction}

A polarized electron-ion collider (EIC) is a planned tool for gluon microscopy to explore the frontiers of quantum chromo-dynamics [1]. The EIC lepton-hadron collider concept is targeting ultra-high luminosities of $10^{34} \mathrm{~cm}^{-2} \mathrm{~s}^{-1}$, approaching those in leptonlepton colliders [2]. In order to deliver such high luminosities, a scheme to use multi-stage electron cooling of the ion beam has been developed by the Thomas Jefferson National Accelerator Facility (JLAB) [3]. In this approach the ion beam is first electron-cooled at low energy to reduce the emittance, and then is accelerated to the collision energy where electron cooling is again continuously reapplied to maintain luminosity during collisions, by suppressing emittance degradation due to non-linear collective effects plus intra-beam and residual gas scattering processes.

However, ionization scattering of the electron beam with residual gas molecules causes ion trapping in the electron rings, both in the collider and electron-cooling system [4]. These trapped ions may cause emittance growth, tune shift, halo formation, and coherent coupled bunch instabilities $[5,6]$. Therefore, the beam's temporal structure needs clearing gaps for timely removal of the residual ions to prevent them from accumulating over many turns. Typically, the gap in the bunch train has a length of a few percent of the net ring circumference [7], so the clearing voltage has to be applied within that interval.

Even with improvements in vacuum technology, ions can fully neutralize the electron beam within seconds for vacuum pressures as low as $10^{-9}$ Torr [8]. Therefore, it is necessary to directly remove the trapped ions in order to avoid or mitigate these unwanted effects. Ion-clearing methods are understood well enough to mitigate ion effects in most storage rings and synchrotrons $[9,10]$. In low repetition rate linacs, ion trapping is typically not 
observed, because the ions have enough time to drift out of the center of the beam pipe between bunches.

With superconducting linacs operating in a continuous wave (c.w.) high current regime [11], the ion trapping is unavoidable [6] and needs to be mitigated. Examples include: strong electron cooling in JLab-proposed EIC [12], Cornell Energy Recovery Linac (ERL) [13], and electron-cooling of ion beam at low energies of 7.7-20 GeV/nucleon in The Relativistic Heavy Ion Collider (RHIC) [14]. With no ion-clearing mechanisms, the ion density would increase until reaching that of the electrons. Therefore, an ion-clearing gap system can be used to reduce trapped ions into the electron beam time structure. In general, such a system includes an in-vacuum electromagnetic deflector (sometimes called "kicker"), a beam dump, and corresponding electronics that provide the waveform of appropriate shape, repetition rate, timing, and magnitude (related to peak and average power).

In response to this problem RadiaBeam, in collaboration with JLAB scientists, is developing an ultra-fast high-voltage (HV) kicker. The system is designed to kick out four of 31 bunches, while keeping the rest in the bunch train, at $43.4 \mathrm{MHz}$ base bunch rep rate to leave a gap of $\sim 92-100 \mathrm{~ns}$ (see Figure 1). This design satisfies the following requirements, summarized in Table 1: $20 \mathrm{mrad}$ deflecting angle at $7 \mathrm{MeV} / \mathrm{c}$ beam momentum, deflector length less than $1 \mathrm{~m}$, gap duration $\sim 100 \mathrm{~ns}$, and $1.397 \mathrm{MHz}$ repetition rate (phase locked). Both the rise and fall time of the deflecting pulses must be less than $20 \mathrm{~ns}(<10 \mathrm{~ns}$ desirable for rise time), with a sub-ns or better timing jitter. The clearing system does not perturb the quality of the beam pulses injected into the linac; it also avoids creating a substantial halo, which must remain $<10^{-5}$ of the beam current. In this paper, we will report the kicker's electromagnetic design, and the initial tests of several high-voltage switches and pulse-forming schemes.

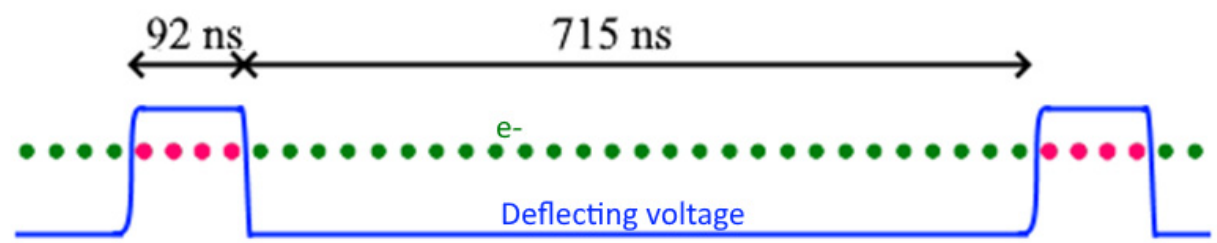

Figure 1. Kicker operation time diagram.

Table 1. Design parameters of a high-voltage kicker.

\begin{tabular}{cc}
\hline Parameter & Value \\
\hline Deflecting angle & $20 \mathrm{mrad}$ (deflecting voltage $140 \mathrm{kV})$ \\
Flange-to-flange length & $50-60 \mathrm{~cm}$ \\
Electron beam energy & $7 \mathrm{MeV}$ \\
Bunch repetition rate & $43.4 \mathrm{MHz}$ \\
Bunch rms transverse size & $\sigma=1 \mathrm{~mm}$ \\
Deflecting pulse width & $92 \mathrm{~ns}$ flat-top required to deflect 4 out of 31 bunches in train \\
Kicker operation per rate & $1.4 \mathrm{MHz}(715 \mathrm{~ns}$ pulsing period) \\
Rise and fall time & $<20 \mathrm{~ns}(10 \mathrm{~ns}$ desired $)$ \\
Aperture (gap) & $14 \mathrm{~mm}$ \\
Required pulsed power & $27.6 \mathrm{~kW}$ peak power per channel $(55.2 \mathrm{~kW}$ total) \\
\end{tabular}

\section{Electromagnetic Design}

Figure 2 demonstrates the electromagnetic model of the kicker, used for the simulations in CST Microwave Studio. The kicker is formed with two parallel electrodes inside a vacuum chamber ( $\varnothing 47.5 \mathrm{~mm}$ pipe matching existing JLAB beamline) and four $50 \Omega$ coaxial ports, two of which serve as power inputs and two are terminated with matching $50 \Omega$ water-cooled loads. The design process was carried out centrally focused around two main aspects: RF behavior and beam dynamics. 


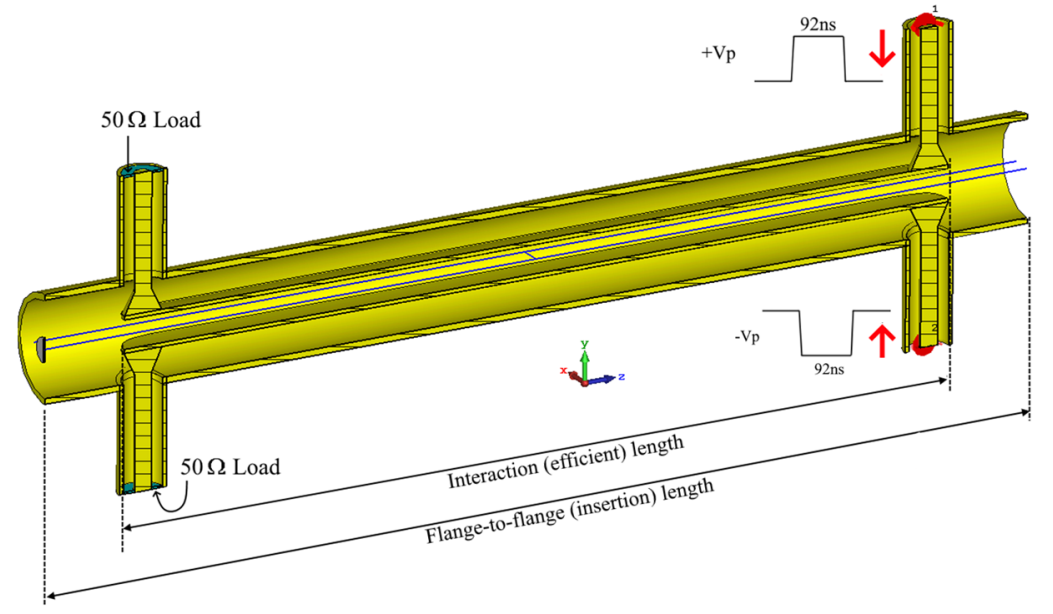

Figure 2. Conceptual design of the stripline traveling wave kicker as a beamline insertion device.

As a four-port RF device, the kicker must be able to provide matching across a bandwidth of at least 0-200 MHz to ensure no distortion in the form of the $10 \mathrm{~ns}$ rise and fall times of 92 ns-long rectangular pulses. This requirement transforms into the following condition: the stripline, consisting of two parallel electrodes inside a pipe, must represent a transmission line with $100 \Omega$ characteristic impedance at an odd mode; given that the structure is excited with $180^{\circ}$ out-of-phase pulses through two $50 \Omega$ inputs. There are two reasons why a $100 \Omega$ impedance is chosen: one is because there is a minimum gap (14 mm) between the electrodes that is driven by the beam rms size $\left(\sigma_{r}=1 \mathrm{~mm}, \pm 6 \sigma_{r}\right)$; the other is simply because it is reasonable to stick with commercially available $50 \Omega$ components, e.g., rigid coaxial lines and high-power loads.

By adjusting the electrodes widths and their curvature radius, we have optimized their shapes to both show $100 \Omega$ characteristic impedance at the odd mode and uniform field around the axis within $\pm 6 \sigma_{r}$ radius $\left(\sigma_{r}=1 \mathrm{~mm}\right)$, as shown in Figure 3. In order to validate how well the impedance-matched kicker conducts the $10 \mathrm{~ns}$ rise times, we ran time-domain simulations while monitoring the pulse shapes at the input ports, in the middle of the structure and at the loads. Simulated waveforms are plotted in Figure 4. From this plot, it is possible to see that the proposed kicker geometry does not distort the operation pulse shape.

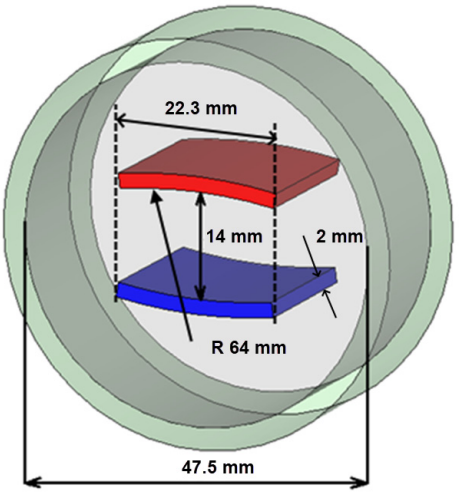

(a)

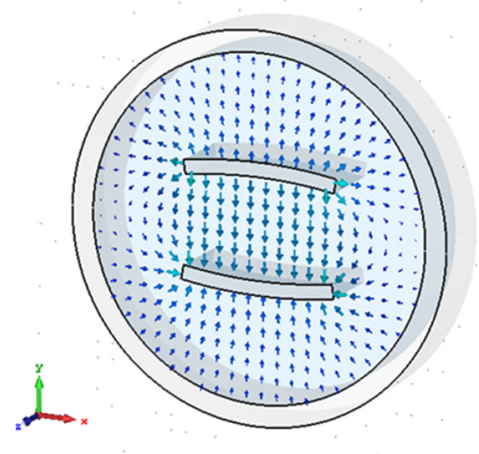

(b)

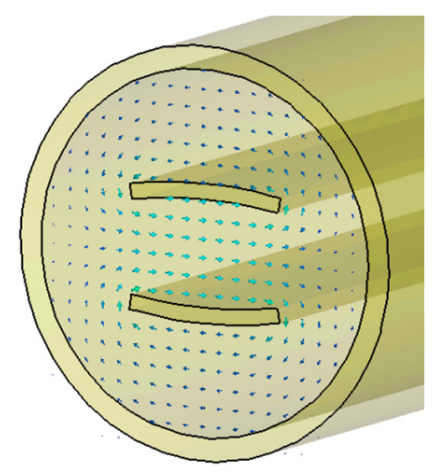

(c)

Figure 3. Transverse profile of the stripline kicker electrodes (a), electric (b) and magnetic (c) field maps. 


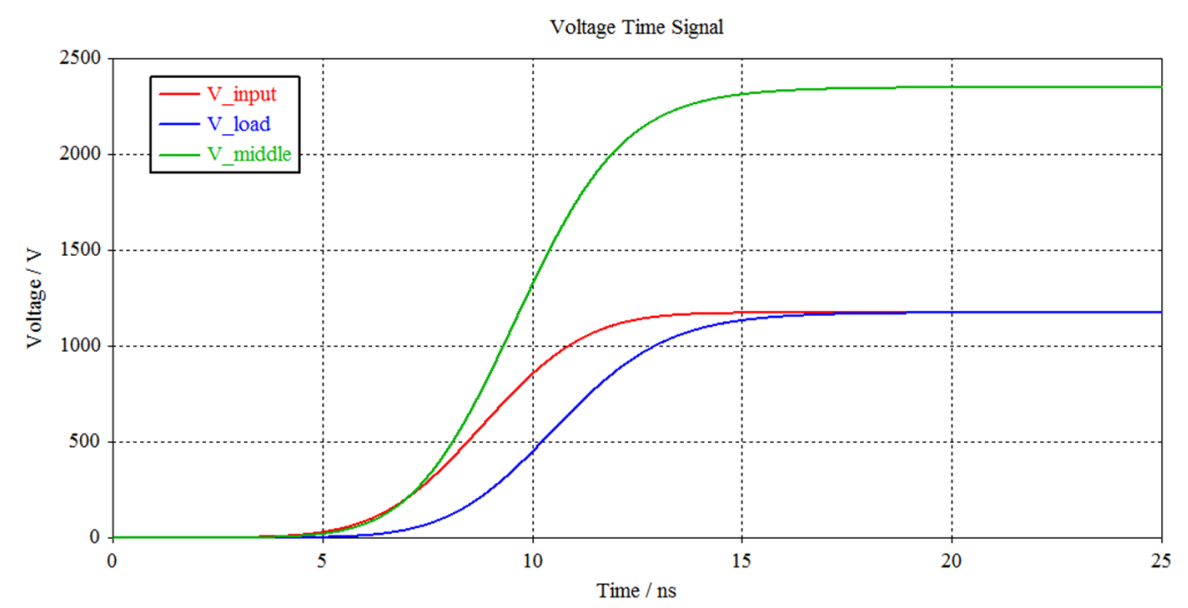

Figure 4. Simulated waveforms propagation: at the input port (red), at the output (blue) and in the middle of the structure for synchronous excitation with two ports (green).

The required deflecting voltage can be calculated using the following expression:

$$
y^{\prime}=\frac{p_{y}}{\sqrt{p_{y}^{2}+p_{z}^{2}}}=20 \mathrm{mrad}
$$

Here $y^{\prime}$ is a deflection angle, $p$ is particle's momentum. For the given $p_{z}=7 \mathrm{MeV} / c$, the results in the required deflection voltage of $V_{y}=140 \mathrm{kV}$. Vertical deflection is provided by both electric $\left(E_{y}\right)$ and magnetic $\left(B_{x}\right)$ components of the field:

$$
V_{y}=\int_{0}^{L_{e f f}} q\left(E_{y}+\beta c B_{x}\right) d z
$$

For $7 \mathrm{MeV}$ electrons their normalized velocity is $\beta=v / c=0.997 . L_{e f f}$ is the kicker effective interaction length. Note, that the two forces will add with one beam direction, and 'cancel' each other with the opposite. The ratio of contributions to the deflection from electric over magnetic forces for a given stripline and loads impedances is $\sim 1: 1$, which gives the estimation of voltage pulse amplitude per port:

$$
V_{p} \approx 140 \mathrm{kV} \frac{1}{2 \times 2} \frac{g}{L_{e f f}}
$$

From this expression we can calculate the total input power, dissipated in two loads, required to provide $20 \mathrm{mrad}$ deflection:

$$
P_{\text {peak }}=2 \times \frac{V_{p}^{2}}{R_{\text {Load }}}=46.8 \times 10^{6}\left(\frac{g}{L_{\text {eff }}}\right)^{2} \mathrm{~W}
$$

Here $R_{\text {Load }}$ is a $50 \Omega$ load impedance and the gap width $(g)$ is considered to be predefined $(14 \mathrm{~mm})$, so the kicker length is the main parameter that will drive the power requirement. The plot in Figure 5 shows the peak and average power consumption dependence on the kicker length. 


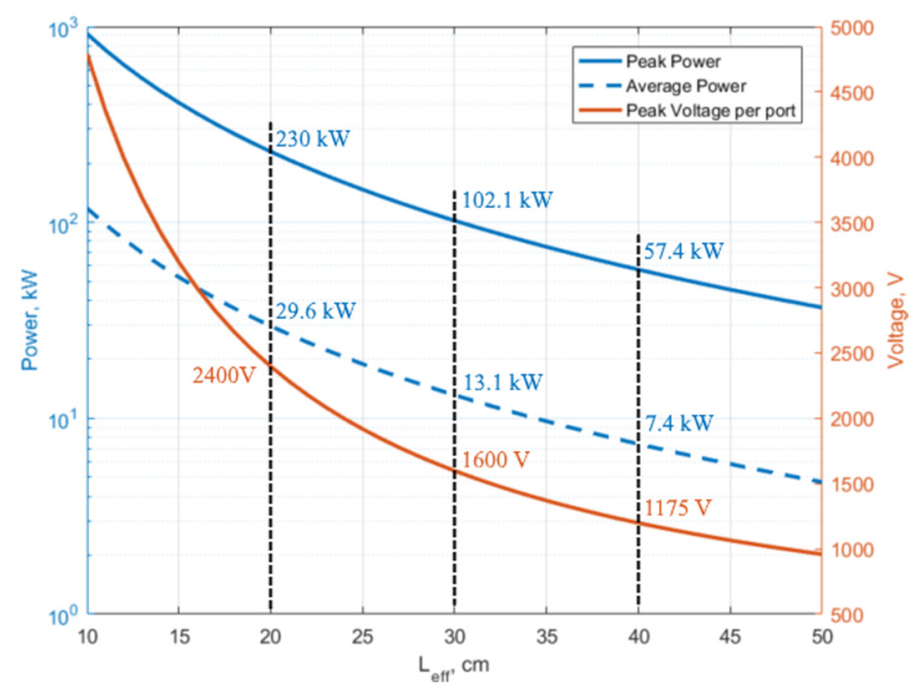

Figure 5. Required voltage and power consumption for $20 \mathrm{mrad}$ deflection in the kicker with $14 \mathrm{~mm}$ gap.

The wakefields of the kicker cavity, which can be excited by the passing electron beam and could cause excessive heat on the cavity wall or degradation of the beam quality, was studied by using CST Particle Studio (wakefield solver). In particular, the heating comes from the trapped modes within the cavity below the cutoff frequency of $\mathrm{TE}_{11}$-mode in the beam pipe, which is about $3.7 \mathrm{GHz}$. The impedance spectrum over the broadband (up to $25 \mathrm{GHz}$ ) is shown in Figure 6 by using fast Fourier transform (FFT) code for accurate evaluation from the wakefields. As shown in this plot, the impedance remains roughly constant over frequency at low values of $10 \sim 100 \Omega$, which would imply about $0.5 \mathrm{~W}$ level of associated heating for JLab EIC operation. It is also expected that this low impedance would translate into minimal effect on beam quality throughout the beam pipe.

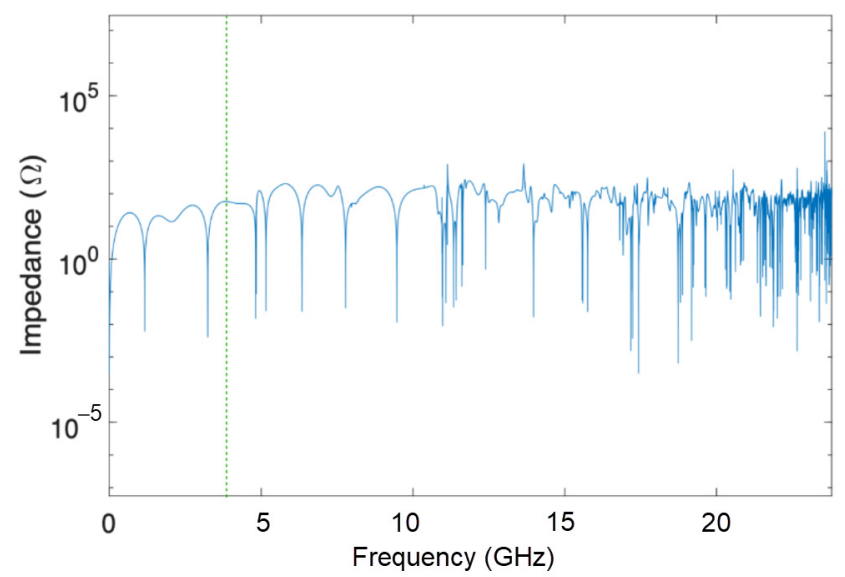

Figure 6. Broadband spectrum of impedance of a stripline kicker cavity. The green dotted line refers to cutoff frequency of the beam pipe.

\section{Beam Dynamics}

To this point, we estimated the required voltage and kicker length from the specified nominal deflection without taking into account the bunch size. In a proposed operation of the stripline kicker cavity at JLAB [15], there is a tight constraint on the beam loss rate due to a relatively large bunch charge. A shorter cavity implies less beam loss but higher $\mathrm{RF}$ power requirements. Therefore, the length of the stripline kicker must be optimized so that the electron bunches go through the cavity without significant beam loss. At the same time, the RF power requirement should be minimized as much as possible. It is also noted 
that the beam loss can be controlled not only by the cavity length but also by the initial injection angle and position of the beam. In practice, the requirement for beam losses is set as less than a couple of $\mathrm{W} / \mathrm{m}$. Therefore, the optimization process is reduced to obtaining the minimum RF power.

In order to find an optimal choice for the kicker cavity length, we consider a series of lengths to check on the beam loss over a range of injection beam parameters. Then, the length would be determined for the case with the lowest corresponding RF power. Since simulating a range of injection parameters directly would have been very time consuming, we instead calculated an analytical description of the beam trajectories and then benchmarked the simulation results by the CST Particle Studio particle-in-cell [16] (PIC) solver for only a few cases. With a reasonable agreement between the two approaches, we found the optimal injection parameters based on the analytical computation and subsequently used the choice to undertake a final simulation in order to predict the beam loss and the RF power of the cavity.

The equation of motion for an electron bunch in y-direction is given as:

$$
\frac{d p_{y}}{d t}=F_{y}
$$

where $F_{y}$ is a Coulomb and Lorentz forces from (2) and can be written as $F_{y}=4 V_{p} / g$ with $V_{p}$ and $g$ being kick voltage and gap width, respectively. The solution within a stripline is trivially obtained with a constant $F_{y}$ :

$$
y^{\prime}=v_{0} / c+C z y=y_{0}+\frac{v_{0}}{c} z+\frac{C}{2} z^{2}
$$

where $C=F_{y} / m \gamma^{2}$, which describes a parabola. With the initial vertical velocity $v_{0}<0$, the tip location of the parabola is given by differentiating Equation (6) with respect to $z$ :

$$
\begin{gathered}
z_{\text {min }}=-\frac{v_{0}}{c} \frac{1}{C} \\
y_{\text {min }}=y_{0}+\left(\frac{v_{0}}{c}\right)^{2} \frac{1}{2 C}
\end{gathered}
$$

The trajectory over a whole kicker cavity, assuming there is no force between the edge of kicker cavity and the stripline is given as:

$$
\begin{aligned}
& y=y_{0}+\frac{v_{0}}{c} z,-l<z<0 \\
& y=y_{0}+\frac{v_{0}}{c} L_{e f f}+\frac{C}{2} L_{e f f}^{2}+\left(\frac{v_{0}}{c}+C L_{e f f}\right)\left(z-L_{e f f}\right), 0<z<L_{e f f}+l
\end{aligned}
$$

where $L_{\text {eff }}$ is the length of stripline and $l$ is the additional length to kicker cavity ends. In particular, the $y$-offset at the exit of the stripline kicker $Y$ is given as:

$$
Y=y 0+\frac{v_{0}}{c} L_{e f f}+\frac{2 e V_{p}}{g m \gamma c^{2}} L_{e f f}^{2}
$$

Now, the distance between the edge of the gap and the center of beam, $(g / 2-Y)$ defines to beam loss $R$ in Gaussian transverse profile of the beam (only on one side) as follows:

$$
R=1-\frac{1}{\sqrt{2 \pi \sigma^{2}}} \int_{-\infty}^{\frac{g}{2}-\Upsilon} e^{-\frac{y^{2}}{2 \sigma^{2}}} d y
$$

where $\sigma$ is transverse $\mathrm{rms}$ size of the beam $(1 \mathrm{~mm})$. This would lead to the beam loss power as:

$$
P_{B L}=Q_{b} f_{b} \frac{W}{e} R
$$


Here $f_{b}, Q_{b}$, and $W$ are, respectively: bunch frequency, bunch charge, and electron energy. For the JLab EIC $Q_{b}=1.6 \mathrm{nC}$ and $f_{b}=43.3 \mathrm{MHz}$, and $W=7 \mathrm{MeV}$, yielding the required powers, provided in Table 1.

The equation of motion (6) for an unkicked bunch becomes trivial with $C=0\left(F_{y}=0\right)$. The offset $Y_{u}$ at the end of the stripline is simply given as $Y_{u}=y_{0}+v_{0} L_{\text {eff }} / \mathrm{c}$ and again the corresponding beam loss $R_{u}$ and power are obtained similarly by (11) and (12). On the other hand, the required power scales as $P=V_{p}^{2} / g$. Here $V_{p} L_{e f f} / g$ is held constant for a fixed deflection angle, leading to $P$ scaling as $\sim 1 / L_{e f f}^{2}$. We avoid changing the gap $(g)$ which will change impedance matching.

The comparison between the theoretical computations and the numerical simulations using the CST Particle is made (see Table 2). For a given set of cavities with lengths of 200-600 $\mathrm{mm}$, the simulations with injection parameters of $0 \mathrm{~mm}$ offset and $0 \mathrm{mrad}$ tilting were undertaken (without space charge effects) and benchmarked by the analytical results. With relatively good agreements between the two, we used analytical evaluation to find optimal injection parameters for minimum beam loss with various cavity lengths. We obtained a spectrum of beam loss over a range of injection parameters-offset and tilting-as shown in Figure 7.

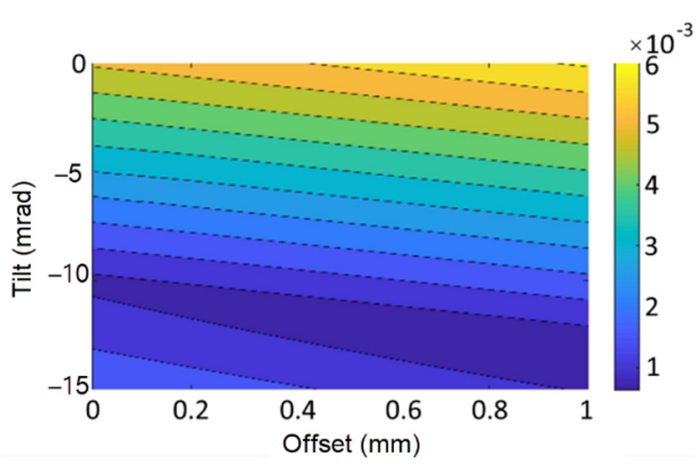

(a)

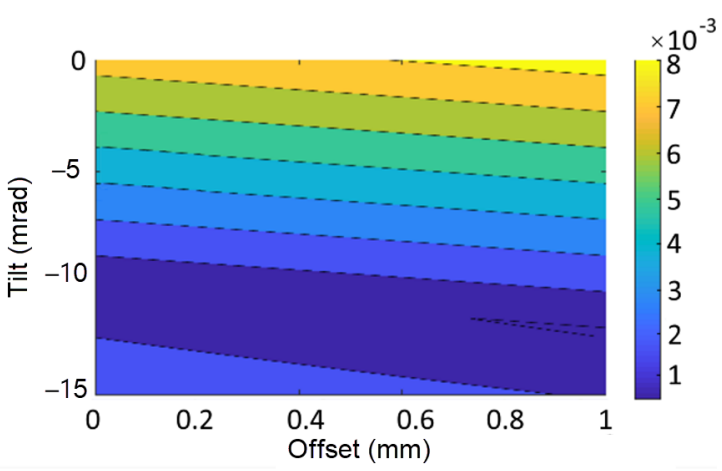

(c)

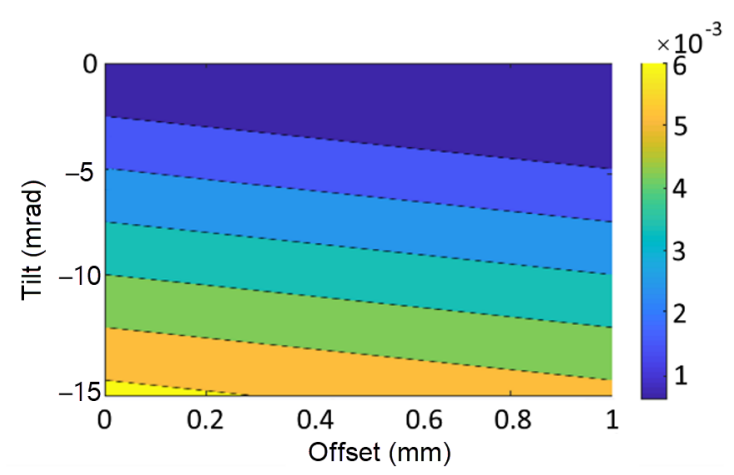

(b)

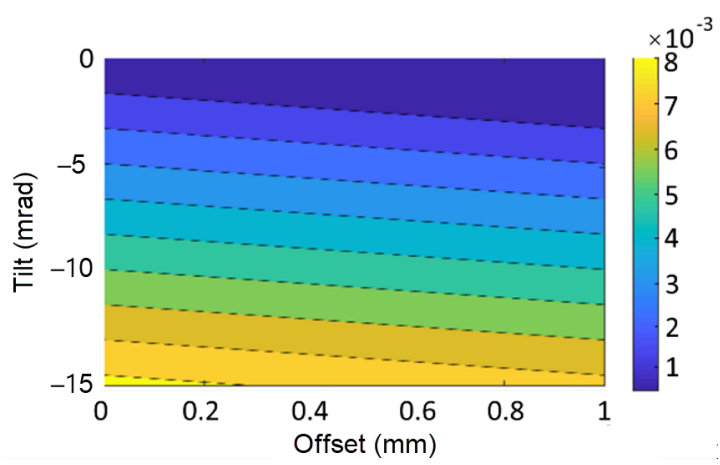

(d)

Figure 7. Exit offset spectrum over injection parameters for kicked $(\mathbf{a}, \mathbf{c})$ and unkicked $(\mathbf{b}, \mathbf{d})$ bunches with the lengths of $400 \mathrm{~mm}(\mathbf{a}, \mathbf{b})$ and $600 \mathrm{~mm}(\mathbf{c}, \mathbf{d})$.

From this study, it is clear that the optimal injection parameters range between $5 \sim 6 \mathrm{mrad}$ tilting and near $0 \mathrm{~mm}$ offset regardless of cavity length. Considering the required pulsed power, it was decided that either $400 \mathrm{~mm}$ or $500 \mathrm{~mm}$ cavity would be close to the optimal choice, i.e., reasonably low RF power with tolerable beam loss, which was confirmed by the CST simulation. Finally, space charge effect was added to give a more realistic prediction on beam loss. This choice would imply the tilting of the kicker cavity by moving the tail of the cavity by about $5 \mathrm{~mm}$ with respect to beam axis, which can be easily controlled. 
Table 2. The beam loss with various strip line lengths.

\begin{tabular}{|c|c|c|c|c|c|c|c|c|}
\hline $\begin{array}{l}\text { Analytical } \\
\text { Evaluation }\end{array}$ & 1 & 2 & 3 & 4 & 5 & 6 & 7 & 8 \\
\hline 20 mrad kick & yes & yes & yes & yes & yes & no & yes & no \\
\hline$L_{e f f}, \mathrm{~mm}$ & 200 & 300 & 400 & 600 & 400 & 400 & 600 & 600 \\
\hline$V_{p}, \mathrm{~V}$ & 2275 & 1560 & 1175 & 790 & 1175 & 1175 & 790 & 790 \\
\hline Offset, mm & 0 & 0 & 0 & 0 & 0 & 0 & 0 & 0 \\
\hline Tilt, mm & 0 & 0 & 0 & 0 & -5 & -5 & -6 & -6 \\
\hline Beam loss, $\%$ & $10^{-6}$ & $8.8 \times 10^{-5}$ & 0.0027 & 0.21 & $5.4 \times 10^{-5}$ & $4.7 \times 10^{-5}$ & $4.6 \times 10^{-3}$ & $4.8 \times 10^{-3}$ \\
\hline$P_{b}, \mathrm{~W}$ & 0.04 & 2.4 & 104.5 & 8100 & 2.1 & 1.8 & 178 & 176 \\
\hline$P_{R F}, \mathrm{~kW}$ & 14.5 & 6.8 & 3.85 & 1.75 & 3.85 & 3.85 & 1.75 & 1.75 \\
\hline \multicolumn{9}{|l|}{ CST simulations } \\
\hline Beam loss, \% & $7 \times 10^{-6}$ & $4 \times 10^{-5}$ & 0.0016 & 0.38 & $4.7 \times 10^{-5}$ & $4.7 \times 10^{-5}$ & $3.6 \times 10^{-3}$ & $3.6 \times 10^{-3}$ \\
\hline$P_{b}, \mathrm{~W}$ & 0.27 & 1.55 & 62 & 14700 & 1.8 & 1.8 & 140 & 140 \\
\hline \multicolumn{9}{|l|}{ Space charge } \\
\hline Beam loss, $\%$ & & & & & $1.8 \times 10^{-3}$ & $4.7 \times 10^{-5}$ & & \\
\hline$P_{b}, \mathrm{~W}$ & & & & & 70 & 1.8 & & \\
\hline
\end{tabular}

\section{High-Voltage Tests}

Our main goal in performing HV modulator experimental work is to test the associated critical components. We consider the switches to be the key element of most pulse-forming devices regardless of their principal schemes. The primary goal of the tests was to confirm that $10 \mathrm{~ns}$ rise and fall times are achievable, the switch is capable of changing its state at the desired 1.4 MHz rep rate with $100 \mathrm{~ns}$ flat-top duration, and that it can conduct high currents without overheating. In order to test the switch, we fabricated a test stripline as shown in Figure 8, so that it mimics one half of the kicker structure. Essentially, the stripline is a $50 \mathrm{~cm}$-long piece of $50 \Omega$ line with $1-5 / 8^{\prime \prime}$ coaxial connectors. We also attached a $50 \Omega$ coaxial load rated to $5 \mathrm{~kW} \mathrm{CW}$ to the output of the stripline and utilized an N-type coaxial adapter to the input.

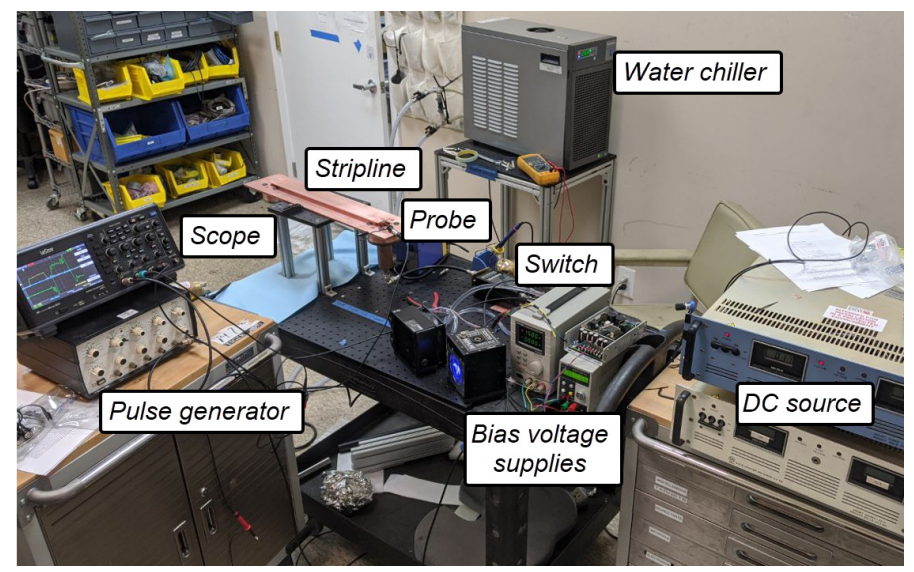

(a)

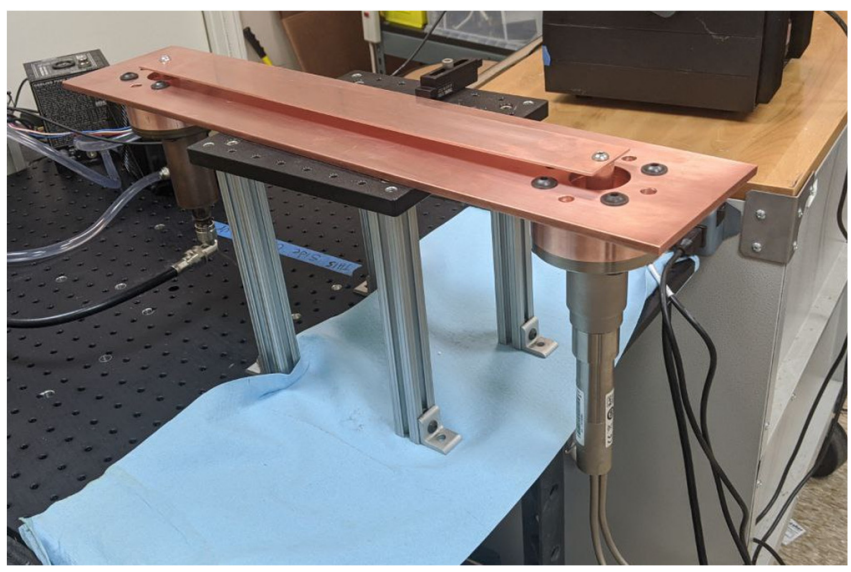

(b)

Figure 8. High-power test stand for high-voltage (HV) switch (a) and a close-up photo of the stripline (b).

First, we performed the reflected power measurement with a vector network analyzer (VNA), which demonstrated the reflection from DC to $1 \mathrm{GHz}$ below $-25 \mathrm{~dB}$. This ensures the acceptance of higher harmonics of the square-wave signal. Rough estimation of the required bandwidth is $5 \times 1$ /rise-time $\sim 500 \mathrm{MHz}$.

The switching circuit is shown in Figure 9. The first switch under test was FSWP-51-02 from Behlke. This is a metal oxide semiconductor field-effect transistor (MOSFET)-based 
switch with internal resistance of $\sim 55 \Omega$. The model is rated to $1 \mathrm{kV}$ at 20 A peak current. The purpose of the tests was to achieve the desired $100 \mathrm{~ns}$ duration, less than $10 \mathrm{~ns}$ rise and fall times and $1.4 \mathrm{MHz}$ pulse repetition frequency (PRF), while gradually increasing the peak voltage and, therefore, the current. In Figure $9, V_{p}$ is a DC power supply, that charges capacitor bank $C_{\text {charge }}$ through a resistor $R_{\text {charge }}$. The capacitor bank is connected to the switch input side, which commutates it to the stripline (50 $\Omega$ input impedance) when the low-voltage pulse is applied to the switch control port.

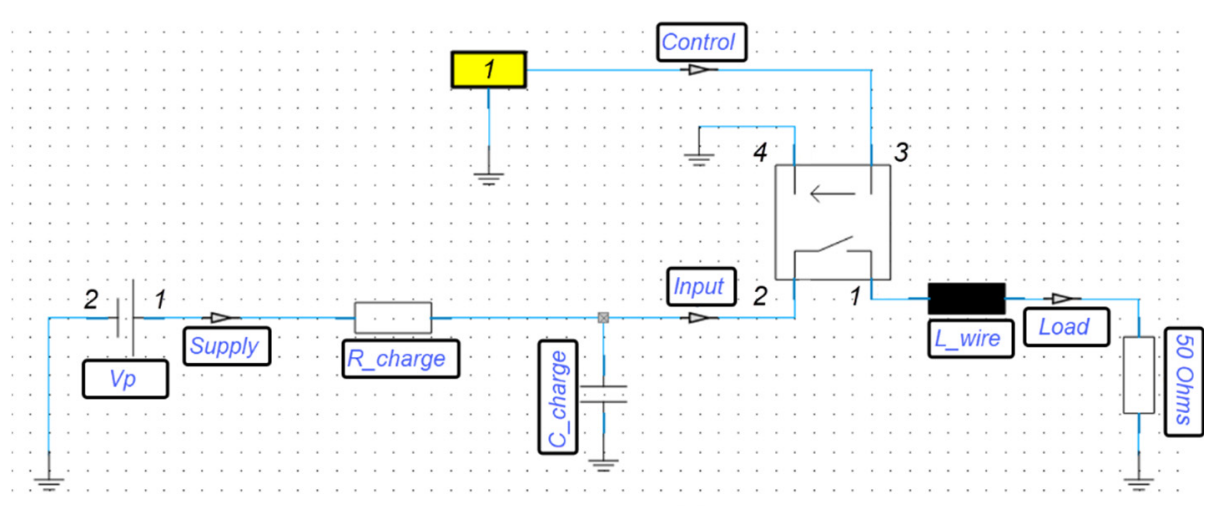

Figure 9. Schematics of a switch test stand: $R_{\text {on }}=55 \Omega, R_{\text {off }}=1 \mathrm{M} \Omega ; R_{\text {charge }}=0.5 \Omega, C_{\text {charge }}=200 \mathrm{nF}$, $L_{\text {wire }}=50 \mathrm{nH}, R_{\text {load }}=50 \Omega$.

The measured waveforms are plotted in the Figure 10. We were able to achieve $6.5 \mathrm{~ns}$ rise and $5.9 \mathrm{~ns}$ fall time with FSWP-51-02. We gradually ramped the supply voltage and the repetition rate and achieved pulse amplitude of $500 \mathrm{~V}(10 \mathrm{~A}$ at the load) and repetition rate of $1.4 \mathrm{MHz}$. At this point, we were too cautious and did not exceed $500 \mathrm{~V}$ peak voltage. We have also noticed that the switch temperature rose to $50{ }^{\circ} \mathrm{C}$ while operating the system at $500 \mathrm{~V}$. This heating corresponds to high intrinsic resistance of the MOSFET $(\sim 55 \Omega)$, which leads to a $50 \%$ loss in the transistor itself.

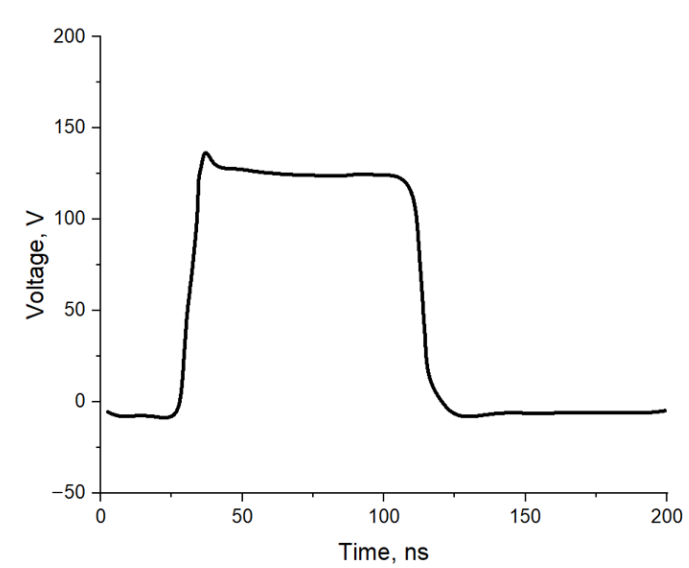

(a)

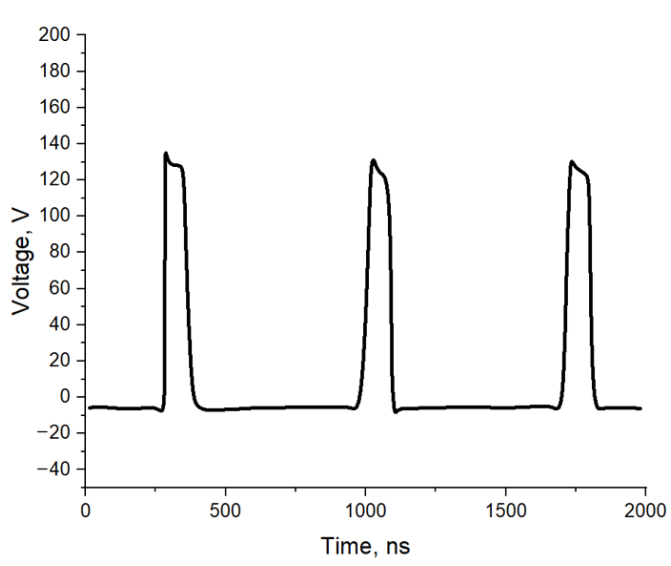

(b)

Figure 10. Measured shape of a single HV pulse with FSWP-51-02 at the load, showing achieved 6.5 ns rise and 5.9 ns fall times (a) and the load voltage pulses, showing achieved 1.4 MHz PRF (b).

While in the process of deciding whether to purchase the second identical FSWP-51-02 switch for testing their combined output, Behkle announced a novel switch FSWP-51-06 based on GaN technology which has $\sim 3 \Omega$ intrinsic resistance and rated to $5.5 \mathrm{kV}$ and $60 \mathrm{~A}$. The main advantage of GaN-based switch as opposed to MOSFET version is in much lower intrinsic losses. We purchased and tested the GaN-based FSWP-51-06 on our HV test stand and successfully achieved $6.8 \mathrm{~ns}$ rise and $4.5 \mathrm{~ns}$ fall times with $1.5 \mathrm{~ns}$ jitter for $410 \mathrm{~V} / 8.2 \mathrm{~A}$ 
pulses at 1.4 MHz PRF with $82 \%$ efficiency, as shown in Figure 11. With such short rise/fall times demonstrated, there is solid basis to expect the GaN-based switch to work on an electron bunching frequency up to $88 \mathrm{MHz}$.

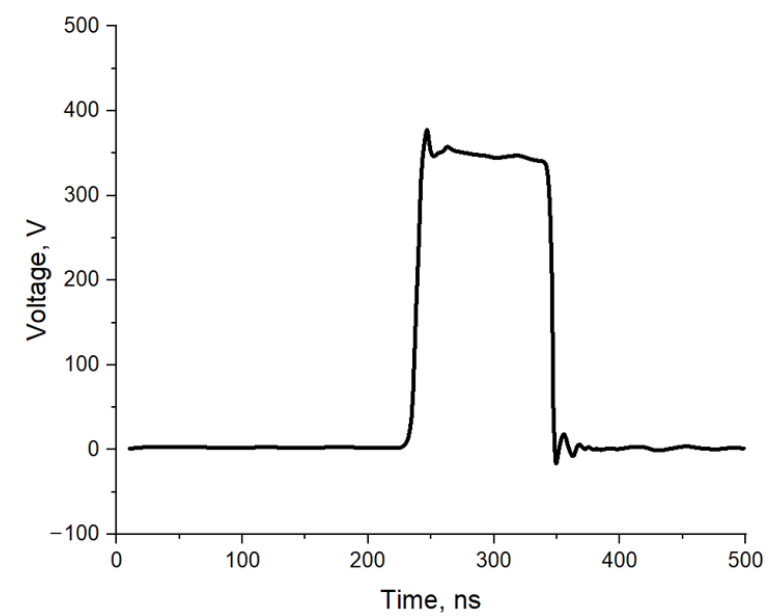

Figure 11. Measured shape of a single 107 ns 410 V/8.2 A pulse from GaN-based FSWP-51-06. Input DC voltage is $500 \mathrm{~V}$.

The next step is to gradually increase the input DC level in order to achieve our goal $1.1 \mathrm{kV} / 22 \mathrm{~A}$ at the output. As GaN performance is dependent on the transmitted power level itself, further testing will be done with a DC supply capable of providing up to $2 \mathrm{kV}$ and $40 \mathrm{~A}$. This exceeds the power for the current application, but slow ramping of its output will experimentally define the requirements on the actual DC source that will be used in the final pulser prototype.

\section{Discussion and Conclusions}

An ion-clearing gap for an electron cooling system is required to operate the next generation of ultra-high luminosity nuclear physics colliders such as the EIC lepton-hadron collider. In this paper, we presented a HV kicker design, capable of achieving an $20 \mathrm{mrad}$ deflecting angle of $7 \mathrm{MeV}$ electron bunch. Based on the optimization of several kicker parameters, we found that its effective length should be $40-50 \mathrm{~cm}$, and the required peak voltage is $1200 \mathrm{~V}$. We showed that solid-state HV switches are capable of providing 92 ns-long pulses at $1.4 \mathrm{MHz}$ rep rate with $10 \mathrm{~ns}$ rise/fall times.

In addition to being an enabling technology for EIC, the proposed system or its constituent blocks may find various uses in HV switching applications, including fast beam choppers [17] for injector test facilities, the next-generation free electron laser (FEL) light sources [18], and inverse Compton scattering gamma ray sources using ERLs [19], ion-clearing gaps for ion accelerators, as well as inverse FEL-driven replacement of radio frequency quadrupole (RFQ) accelerating structures [20].

Industrial and defense applications include multi-kW drivers of Pockels cells for high average power lasers, heat management of high-power Class-F [21] microwave solid state power GaN amplifiers [22], high-power DC-DC and AC-DC convertors, directed energy broadband systems, geo-radars employed in geophysics, and high-power military electronics.

Author Contributions: Conceptualization, A.V.S. and S.Z.; methodology, A.Y.S.; software, G.T.P., J.G., H.W. and S.Z.; validation, A.Y.S. and D.G.; formal analysis, A.Y.S. and D.G; investigation, A.Y.S., R.A., A.M., S.V.B., G.T.P., J.G., H.W. and S.Z.; writing—original draft preparation, S.V.K., A.Y.S. and S.Z.; supervision, S.V.K.; project administration, A.Y.S.; funding acquisition, S.V.K., A.Y.S. All authors have read and agreed to the published version of the manuscript.

Funding: This work is supported by the U.S. Department of Energy, Office of Science, Office of Nuclear Physics under SBIR grant DE- SC0019719 and contract DE-AC05-06OR23177. 
Institutional Review Board Statement: Not applicable.

Informed Consent Statement: Not applicable.

Data Availability Statement: Data available from the authors upon reasonable request.

Conflicts of Interest: The authors declare no conflict of interest.

\section{References}

1. Accardi, A.; Albacete, J.L.; Anselmino, M.; Armesto, N.; Aschenauer, E.C.; Bacchetta, A.; Boer, D.; Brooks, W.K.; Burton, T.; Chang, N.-B.; et al. Electron-Ion Collider: The next QCD frontier. Eur. Phys. J. A 2016, 52, 268. [CrossRef]

2. Zimmermann, F. Future colliders for particle physics-“Big and small”. Nucl. Instrum. Meth. Phys. Res. A 2018, 909, 33-37. [CrossRef]

3. Zhang, H.; Zhang, Y.; Derbenev, Y.; Benson, S.; Roblin, Y. Multi-Stage Electron Cooling Scheme for JLEIC. In Proceedings of the 9th International Particle Accelerator Conference (IPAC'18), Vancouver, BC, Canada, 29 April-4 May 2018; pp. 397-399.

4. Thieberger, P.; Altinbas, Z.; Carlson, C.; Chasman, C.; Costanzo, M.; Degen, C.; Drees, K.A.; Fischer, W.; Gassner, D.; Gu, X.; et al. High energy Coulomb-scattered electrons for relativistic particle beam diagnostics. Phys. Rev. Accel. Beams 2016, $19,041002$. [CrossRef]

5. Mether, L.; Oeftiger, A.; Rumolo, G. Modeling of fast beam-ion instabilities. CERN Yellow Rep. Conf. Proc. 2018, 1, 63-68. [CrossRef]

6. Hoffstaetter, G.H.; Liepe, M. Ion clearing in an ERL. Nucl. Instrum. Meth. Phys. Res. A 2006, 557, 205-212. [CrossRef]

7. Chao, A.W.; Mess, K.H.; Tigner, M.; Zimmermann, F. Handbook of Accelerator Physics and Engineering, 2nd ed.; World Scientific Publishing Co.: Hackensack, NJ, USA, 2013.

8. Full, S.; Bartnik, A.; Bazarov, I.V.; Dobbins, J.; Dunham, B.; Hoffstaetter, G.H. Detection and clearing of trapped ions in the high current Cornell photoinjector. Phys. Rev. Accel. Beams 2016, 19, 034201. [CrossRef]

9. Poncet, A. Ion trapping, clearing, beam-ion interactions. In Proceedings of the CERN Accelerator School Course on Vacuum Technology for Particle Accelerators, Copenhagen, Denmark, 28 May-3 June 1999; pp. 165-202.

10. Dienes, M.; Month, M.; Strasser, B.; Turner, S. Frontiers of Particle Beams: Factories with e+ e- Rings. Lect. Notes Phys. 1994, 425, 202-221. [CrossRef]

11. Ben-Zvi, I. Superconducting energy recovery linacs. Supercond. Sci. Technol. 2016, 29, 103002. [CrossRef]

12. Zhang, H.; Roblin, Y.; Zhang, Y.; Derbenev, Y.; Benson, S.; Li, R.; Chen, J.; Huang, H.; Luo, L. Simulation Study on JLEIC High Energy Bunched Electron Cooling. In Proceedings of the North American Particle Accelerator Conference 2016, Chicago, IL, USA, 9-14 October 2016; pp. 568-571.

13. Hajima, R. Energy Recovery Linacs for Light Sources. Rev. Accel. Sci. Technol. 2012, 3, 121-146. [CrossRef]

14. Gassner, D.M.; Fedotov, A.; Hulsart, R.; Kayran, D.; Litvinenko, V.; Michnoff, R.; Miller, T. Instrumentation for the Proposed Low Energy RHIC Electron Cooling Project with Energy Recovery. In Proceedings of the 3rd International Beam Instrumentation Conference 2014, Monterey, CA, USA, 14-18 September 2014; pp. 49-54.

15. Tennant, C.; Alarcon, R.; Balewski, J.; Benson, S.; Bernauer, J.; Bessuille, J.; Blyth, D.; Boyce, J.; Cao, T.; Cervantes, R. LERF-New Life for the Jefferson Lab FEL. In Proceedings of the ERL 2017, the 59th ICFA Advanced Beam Dynamics Workshop on Energy Recovery Linacs, Geneva, Switzerland, 18-23 June 2018; pp. 45-48. [CrossRef]

16. Hockney, R.W.; Eastwood, J.W. Computer Simulation Using Particles, 1st ed.; CRC Press: Boca Raton, FL, USA, 1988; ISBN 9780852743928.

17. Novikov-Borodin, A.V.; Kutuzov, V.A.; Ostroumov, P.N. A fast chopper for intensity adjustment of heavy-ion beams. In Proceedings of the IEEE Particle Accelerator Conference (PAC'05), Knoxville, TN, USA, 16-20 May 2005; Volume 2005, pp. 1692-1694.

18. Couprie, M.E. New generation of light sources: Present and future. J. Electron. Spectrosc. Relat. Phenom. 2014, 196, 3-13. [CrossRef]

19. Drebot, I.; Bacci, A.; Bosotti, A.; Broggi, F.; Canella, F.; Cardarelli, P.; Cialdi, S.; Faillace, L.; Galzerano, G.; Gambaccini, M.; et al. BriXs Ultra High Flux Inverse Compton Source Based on Modified Push-Pull Energy Recovery Linacs. Instruments $2019,3,49$. [CrossRef]

20. Avreline, N.; Kutsaev, S.V.; Avreline, A.; Murokh, A. Acceleration of heavy ions in inverse free electron laser. Laser Phys. Lett. 2021, 18, 055402. [CrossRef]

21. Raab, F.H. Class-E, class-C, and class-F power amplifiers based upon a finite number of harmonics. IEEE Trans. Microw. Theory Tech. 2001, 49, 1462-1468. [CrossRef]

22. Smirnov, A.; Agustsson, R.; Ahmadi, M.; Blanchard, P.; Boucher, S.; Branner, R.; Hartzell, J.; Hoyt, K.; Khodos, V.; Mccann, D. Progress on 1.5 GHz Multi-kW CW Amplifier. In Proceedings of the 9th International Particle Accelerator Conference (IPAC'18), Vancouver, BC, Canada, 29 April-4 May 2018. 